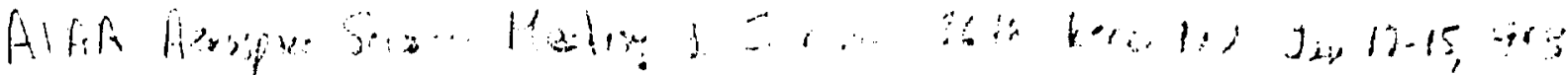

$$
\begin{aligned}
& \text { Copyright } \odot 1997 \text {, American Institute of Aeronautics and Astronautics, Inc. }
\end{aligned}
$$

AIAA-98-0570

\title{
BUOYANCY AND PRESSURE EFFECTS ON BULK METAL-OXYGEN REACTIONS
}

\author{
A. Abbud-Madrid*, C. McKnight ${ }^{\dagger}$, M. C. Branch ${ }^{\ddagger}$, and J. W. Daily ${ }^{\S}$ \\ Center for Combustion and Environmental Research \\ Department of Mechanical Engineering \\ University of Colorado at Boulder \\ Boulder, $\mathrm{CO} 80309-0427$
}

\section{Abstract}

The combustion behavior of metal-oxygen reactions in a weakly broyant environment is studied to understand the rate-controlling mechanisms in the homogeneous and heterogeneous combustion of bulk metals. Cylindrical titanium and magnesium specimens are ignited in pure-oxygen at pressures ranging from 0.1 to $4.0 \mathrm{~atm}$. Rechuced gravity is obtained from an aircraft thing parabolic trajectories. A weakdy buoyant enviromment is generated at low pressures under normal gravity and also at $1 \mathrm{~atm}$ under reduced gravity $(0.01 \mathrm{~g})$. The similarity between these two experimental conditions comes from the $p^{2} g$ buoyancy scale extracted from the Grashof number. Lower propagation rates of the molten interface on titanium samples are found at progressively lower pressures at $1 \mathrm{~g}$. In the reduced-gravity experiments at $1 \mathrm{~atm}$, the propagation velocity is almost half the value obtained at $1 \mathrm{~g}$. These rates are compared to theoretical results from heat conduction analyses with a diffusion/convection controlled reaction. The close agreement found between experimental and theoretical values indicate the importance of natural convectionenhanced oxygen transport on combustion rates. For magnesium, progressively longer burning times are experiencod at lower pressures and $1 \mathrm{~g}$. Under reduced gravity conditions at $I$ atm, a buming time twice as long as in $1 \mathrm{~g}$ is exhibited. However, in this case, the validity of the $p^{2} g$ buoyancy scale remains untested due to the inability to obtain steady gas-phase burning of the magnesium sample at $0.1 \mathrm{~atm}$. Nevertheless, longer burning times and larger flame standoff distances at low pressures and at low gravity points to a diffusion/convection controlled reaction.

\footnotetext{
- Research Associate, Member AlAA

+ Gradnate Student

† Professor, Member AIAA

$\S$ Professor, Associate Fellow AIAA
}

Copyright $\Theta 1998$ by the American Institute of Aeronautics and Astronautics, Inc. All rights reserved.
Introduction

Reactions between metals and gaseous species are important in a number of practical systems. These include: rocket propellants with metal particles added for increased thrust and suppression of combustion instability, the self-propagating high-temperature synthesis processes (SHS) to produce new high temperature materials, the combustion of metal fuels for planetary missions, and vapor-deposition processes. In spite of the many practical applications, the combustion of metals remains an incompletely understood phenomenon due to the interaction of the complex processes involved, including lowtemperature surface oxidation, oxidizer diffusion, heterogeneous reactions, product condensation, highemissivity of products, natural convection, and multiphase thermodynamics and reaction kinetics.

As bas been the case with many other combustion phenomena, conducting metal combustion experiments in the absence of gravity may provide further understanding of the complex physical and chemical mecharisms involved. A microgravity environment reduces the disturbing buoyant effects that frequently obscure weaker forces such as molecular diffusion. At the same time this type of environment permits detailed, longer-duration observations in largescale experiments which are usually inaccessible due to the rapid development of buoyancy-driven disturbances. The use of subatmospheric pressures can also reduce the effect of natural correction and allow larger spatial and temporal seales for flame studies.

Gravity and pressure effects on buoyancy induced convection are linked through the Grashof number $(G r)$ which evaluates the relative importance of molecular and buoyant transport. The Grashof number is defined as:

$$
G r=\frac{(\Delta \rho / \rho) g L^{3}}{v^{2}}
$$

where $\Delta p$ is the characteristic density difference, $\rho$ is the mean density, $g$ is the acceleration of gravity, $L$ is

This is a preprint or reprint of a paper intended for presentation at a conference. Because changes may be made before formal publication, this is made available with the understanding that it will not be cited or reproduced without the permission of the author. 
the characteristic length scale, and $v$ is the kinematic viscosity. Extracting the gravity $(g)$ and pressure $(p)$ dependence from $\mathrm{Eq}$. (1) results in the $p^{2} g$ buoyancy scale'. The use of low pressures at normal gravity to reduce the effect of buoyancy has been employed in the study of several flame configurations ${ }^{i A}$.

In the case of metals the $p^{2} g$ buoyancy scale can be used to assess the importanco of gas diffinsion as a buming-rate controlling mechanism. Several mechanisms have been identified" as being ratecontrolling at metal burning temperatures including: a) oxygen diffusion/convection to the sample surface, b) physical and chemical adsorption of oxygen molecules and atoms at the oxygen-oxide boundary, c) diffirsion of jons through the oxide layer, and d) reaction at the oxide-metal boundary. The pressure dependency varies for each of these processes. Conducting experiments at various gravity levels while keeping the pressure constant has the advantage of assessing only the role of oxygen diffusion and convection on the burning rate. On the other hand, experiments at various pressures at normal gravity may be used to correlate the burning rates obtained under both sets of experimental conditions. While many investigations ${ }^{6,9}$ have studied the effect of pressure on metal combustion only a few studies bave evaluated the influence of gravity on the burning behavior of metals ${ }^{\text {fin }} 10$.

By conducting experiments at rednced gravity and constant pressure and at various pressures at normal gravity, the present investigation provides new insight into the possible role of oxygen diffusion/convection as a rate-controlling mechanism in the burning of bult metals in pure oxygen. Titanium (Ti) and magnesium (Mg) metals are chosen to represent the two different combustion modes observed in metals: beterogencous, multi-phase surface combustion (as in Ti) and homogeneous, gas-phase combustion (as in Mg). Metal surface temperatures, propagation mtes, burning times, and spectroscopic measurements are obtained under normal and reduced gravity. Reduced gravity is obtained from an aircraft flying parabolic trajectories. Visual evidence of all phenomena is provided by high-speed cinematography.

\section{Experimental System}

The apparatus and experimental procechures nsed in this investigation have been thoroughly described previously ${ }^{10}$ in , bence only a brief description will be given here. A schematic of the experimental system is shown in Fig. 1.

The ignition source consists of a $1000-W$ xenon lamp that generates a highly collimated beam
( $4^{\circ}$ half angle) with broadband radiation (300-1100 nm). An aspheric leas focuses the beam to provide a $2-\mathrm{MW} / \mathrm{m}^{2}$ power density on the top surface of a 4-mmdiameter and 4-mm-high metal specimen that sits on an alumina holder. A 4.5-L, stainless steel, cylindrical combustion vessel houses the lens, metal specimen, and alumina holder. Optical access for the movie amera and spectrograph is provided through two fused-silica side windows, while a thind window is used for sample replacement. $\mathrm{Ti}$ and $\mathrm{Mg}$ metal specimens (99.95\% purity) are burned in an $O_{2}$ environment $(99.6 \% \mathrm{~min}$ ) at pressures varying from 0.1 to $4 \mathrm{~atm}$. The surface temperature is measured with a $0.125-\mathrm{mm}$ diameter, type $R$ thermocouple attached to the outer wall of the sample.

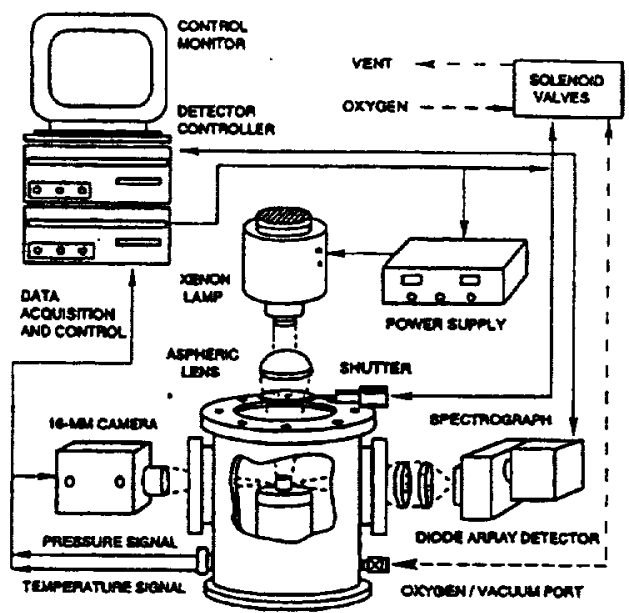

Migure 1. Diagram of the experimental system.

A high-speed, 16-mm movie camera provides surface and flame vispalization; the images are also used for measurement of propagation rates and burning times. With a $7.5^{\circ}$ shutter and speeds up to 500 frames/s, exposure times $x$ short as 1/20,000 s are obtained. In addition to virible light imaging, timoand space-resolved spectral information on gas-phase reactants and products is obtained with an imaging spectrograph and a 1024-element diode array detectar.

The experiment is controlled entirely by a computer, a digital/analog data acquisition board, and an internoce code written in graphical programming software. The recuced-gravity experiments were conducted onboard the NASA-Lewis DC-9 Research Aircratt in Cleveland, Ohio. Up to $20 \mathrm{~s}$ of reduced 
gravity $( \pm 0.01 \mathrm{~g})$ were available in a single parabolic maneurver. A minimum of ten tests were conducted for each metal at the various pressures and gravity levels to ensure experimental repeatability.

\section{Results and Discussion}

The role of oxygen diffusion/convection on the burming rates of $\mathrm{Ti}$ and $\mathrm{Mg}$ is evaluated by two sets of experiments. The results form the reduced-gravity tests at a constant pressure are discussed first followed by the resuits from the tests at various pressures at normal gravity. A comparison of the observations from the two experiments is attempted by using the $p^{2} g$ buogancy scale.

\section{Reduced Gravity Effects}

\section{a) Titanium}

For $T i$ the critical temperature $\left(T_{\text {eril }}\right)$ of the samplo- the temperature at which the heat generated by oxidation first overcomes beat losses, leading to igmition-accurs at a point on the top $\mathrm{nim}$ of the cylinder at around $1750 \mathrm{~K}$ Afterward, a molten mass consisting of a mixture of metal and oxides starts traveling across the upper surface of the specimen. Under normal or reduced gravity conditions, steady downwand propagation of a spherical mass follows in a smooth, nonexplosive fashion until reaching the almmina base. No evidence of emission from gaseous species is found from the visible images taken by the high-speed camera. The lack of any significant vaporphase reactions during this stage of the combustion process (firther verified by spectroscopic measurements), points to the existence of a predominantly beterogeneous reaction with condensed products in the liquid and solid surfaces of the specimen.

Figure 2 shows a graph of the vertical distance traveled by the molten surface as a function of time for typical samples at $1 \mathrm{~g}$ and low $\mathrm{g}$. The propagation velocities inferred from the slope of the fitted lines are $16.2 \mathrm{~mm} / \mathrm{s}$ and $8.7 \mathrm{~mm} / \mathrm{s}$ under normal and $10 \mathrm{w}$ gravity. Similar velocities were obtained for all the samples tested (with standard deviations of $0.6 \mathrm{~mm} / \mathrm{s}$ and $0.3 \mathrm{~mm} / \mathrm{s}$ for the $1-g$ and the $10 w-g$ cases, respectively).

Evaluating the ratio of propagation velocities at normal $\left(V_{n}\right)$ and low ( $\left.V i\right)$ gravity gives a value of 1.86. A steady propagation behnvior during this period at $1 \mathrm{~g}$ suggests that the propagation velocity has not been altered by the influence of the gravity force on the molten mass-also confirmed by the spherical shape of the reacting mass in the high-speed photographs. Furthermore, a calculation of the Bond mumber $(B O=$ gravitational force/surface tension force) under these conditions results in values below 0.1 . For large values of the Bond number $(B o>1)$, acceleration can occur by lateral dripping when the gravitational force overcomes the surface tension force holding the molten mass together.

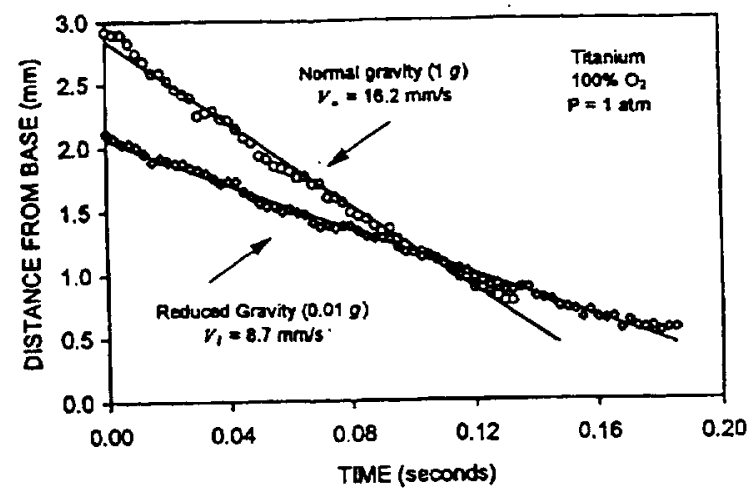

Figure 2 Propagation of the reacting molten mass in $T i$ samples under normal and rectuced gravity at 1 atm.

Following a theoretical approach similar to the one used for gaseous flame propagation, several studies, 12 have obtained an expression of the form $V \sim(w)^{1 / 2}$ to calculate the propagation velocity $V$ along metal cylinders undergoing heterogeneous surface burning with a rate of reaction $w$. Considering the diffusion and convection of $\mathrm{O}_{2}$ to the sample as the rate-limiting step, the reaction rate becomes proportional to $w-(G r)^{1 / 4}$, where $G r$ is the Grashof number. The propagation velocity then depends on $G r$ as $V-(G r)^{1 / n}$, so that $V-(g)^{1 / n}$. Evaluating $V / V_{l}$ for the normal $(1 \mathrm{~g})$ and low gravity $(0.01 \mathrm{~g})$ cases, a theoretical ratio of 1.78 is obtained The close agreement between experimental (1.86) and theoretical (1.78) ratios of propagation velocities indicates the importance of the influence of natural-convectionenhanced oxygen transport on combustion rates.

This result disagrees with findings from studies that suggest other rate-controlling mechanisms for metals exhibiting heterogenoous combustion. Among these proposed mechanisms are: a) the incorporation of oxygen into the oxide layers, physical and chemical adsorption of oxygen on the oxide surface ${ }^{13}$, and c) the mixing of oxygen with the metal 
in the molten ball ${ }^{14}$. The first two mechanisms may control the burning rate in the presence of a continuous, coherent oxide layer which separates the metal from the oxygen. Based on the high-speed movie images recorded in the present investigation, non- $\infty$ herent, irregular patches of liquid oxides are clearly seen floating and moving on the molten ball. Even though no reliable information is currently available on adsorption rates of oxygen through liquid metals and oxides, adsorption rates in the liquid state should be significantly higher than in solids since liquid oxides are not considered effective diffusion barriers's.

A poor mixing of metal and oxygen would occur in the case of slow circulation of material in the molten ball. In the aforementioned burning $\mathrm{T} i$ samples strong circulation currents are observed in the molten surface. This circulation is caused, for the most part, by surface tension driven flow in a region with a pronounced temperature gradient-an additional small contribution may also come from unstable density gradients in the normal gravity case. An estimate of the magnitude of this surface tension driven internal flow is possible by evaluating the Marangoni number, $M a$, which considers the relative importance of surace tension and viscous forces. A detailed evaluation " of this number based on experimental measurements and thermophysical data of liquid $\mathrm{Ti}$ gives a value of $\mathrm{Ma}=$ 6931. Since a Marangoni number above the critical value $\mathrm{Ma}_{\mathrm{crit}}=80$ is required for the onset of a surfacetension-induced hydrodynamic instability', the calculated value indicates a strong flow circulation inside the molten mass. This circulation allows for an efficient adsorption of oxygen and a thorough and rapid mixing of reactants. Thus, according to the results of the low-gravity experiments, the ratecontrolling mechanism in the combustion of bulk $\mathrm{Ti}$ samples appear to be the diffusion/convection of oxygen to the sample surface.

\section{b) Magnesinm}

In the case of $\mathrm{Mg}$, a critical temperature of $1050 \mathrm{~K}$ is obtained. Following the first flash generated by the $\mathrm{Mg}^{-\mathrm{O}_{2}}$ vapor-phase bomogenocus reaction, an ignition wave runs through the sample driven by the difference between the flame temperature (near 3430 $\mathrm{K}$, the vaporization-decomposition point of $\mathrm{MgO}^{17}$ ) and the temperature of the unreacted metal, which is near the $\mathrm{Mg}$ boiling point (1366 K). Owing to the irregularly shaped (a consequence of metal melting before ignition), porous, solid oxide layer surrounding the sample, no attempt was made to calculate surface regression rates. Instead, an evaluation of burning times and a qualitative discussion of important phenomena is given.

From the visible images and emission spectra measurements, the structure of the luminous flame that engulfs the sample after the passage of the ignition wave is in general agreement with the extended reaction zone model of Glassman et $\mathrm{al}^{12}$. According to this model, an inner region of $\mathrm{Mg}-\mathrm{O}_{2}$ vapor-phase reaction is followed by MgO condensation; the solid MgO particles eventually pile up in the bright white flame front captured in the high-speed photographs.

During fully developed combustion, buoyancygenerated convection currents are responsible for the main differences observed in the two gravity cases investigated. At $1 \mathrm{~g}$, high convection currents forming an elongated vertical plume enhance burning by increasing $\mathrm{O}_{2}$ flux to the reaction zone and by removing oxide products that may constitute a barrier to $\mathrm{O}_{2}$ diffusion. The proximity of the resulting flame front to the metal sample is an indication of fast burning rates. In comparison, at low gravity conditions, the severe reduction of convectionthreefold, according to the $(G r)^{1 / 4}$ dependenco-and the increased resistance to $\mathrm{O}_{2}$ diffusion by combustion products diminish the oxygen transport. Thus in the reduced-gravity case a broader, outer blue zone (of stagnant oxide particles in the absence of convection curreats) and an increased flame standoff distance from the metal core is detected in the high-speed photographs as compared to the $1-\mathrm{g}$ case. The flame frout diameter increases to accommodate greater oxygen flux and maintain the stoichiometry. Burning times vary widely depending on the number of jets and explosions that accelerate combustion. Nevertheless, the average burning time at low gravity $(3.9 \mathrm{~s})$ is almost twice the average value at $1 \mathrm{~g}(2.2 \mathrm{~s})$. A similar effect of low gravity on burning rates of gas-phase diffusion reactions have been observed in many other flame configurations 19 .

\section{Pressure Effects}

\section{2) Titanium}

The effect of pressure on propagation rates of Ti bulk samples is evaluated by experiments conducted at normal gravity at pressures in the 0.1 to $4.0 \mathrm{~atm}$ range. The test at $0.1 \mathrm{~atm}$ is intended to replicate the weakly buoyant condition experienced in the reduced gravity aircraft at $0.01 \mathrm{~g}$. The similarity of experimental conditions comes from the $p^{2} g$ buoyancy scale described in the Introduction.

The results from the series of pressure experiments are shown in a plot of propagation rate vs. 
$\mathrm{O}_{2}$ pressure (Fig. 3). The trendline in the figure shows a linear variation of the propagation rate with the square root of the oxygen pressure. This result agrees with previous propagation rato-pressure relationships obtained by several research groups with various metals ${ }^{6}$. The decreasing trend in propagating rates at progressively lower pressures appears to indicate the existence of a diffusion/convection controlled reaction. The close agreement of the mean propagation velocities at $0.01 \mathrm{~g}$ and $1 \mathrm{~atm}(8.7 \mathrm{~mm} / \mathrm{s})$ and at 0.1 atm at normal gravity $(7.5 \mathrm{~mm} / \mathrm{s})$ seem to confirm this hypothesis. Care mnst, however, be exercised in assigning the control of the burming rate to a single mechanism at these low pressures. Accorting to Hirano et al." chemical adsorption of oxygen atoms into the molten oxide also follow a square root dependency with pressure. Although a contimuous, coberent oxide layer surrounding the molten ball is not observed in the burning $T i$ samples, several rate-controlling mechanisms may be competing at low subatmospheric pressures.

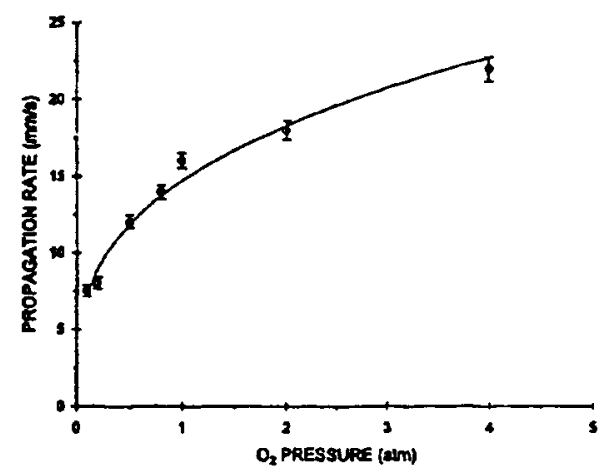

Figure 3. Propagation rates of the reacting molten mass in Ti samples at normal gravity under various oxygen pressures.

Tests at lower-than-0.1 atm pressures were attempted with no evidence of a combustion event. It is believed that an ignition limit rather than a flammability limit is reached at these low pressures. Due to the reduced convection of oxygen, the surface oxidation of the solid metal prior to ignition may switch from a kinetic- to a diffusion-controlled reaction. At a low rate of heat generation by oxidation as compared to the high rate of the varions beat losses, a critical temperature is never reached. Instead, an increasingly thicker, refractive oxide layer is slowly formed resulting in the inability of the lamp to heat up the sample above its conduction and radiation losses. After several seconds of heating, a steady temperature less than a $100 \mathrm{~K}$ lower than the critical temperature is recorded by the thermocouple attached to the metal surface.

\section{b) Magnesium}

The same set of experiments at pressures from 0.1 to $4.0 \mathrm{~atm}$ were conducted with $\mathrm{Mg}$. For the reasons explained previously, the burning time of the $\mathrm{Mg}$ samples is measured instead of the burning rate of an irregularly shaped molten specimen. The buraing times follow the same trend with pressure as observed in the propagation rates of $\mathrm{Ti}$ samples. Shorter burning times characterized by sharply elongated flames occur at progressively higher pressures. Longer burning times and broader flames are exhibited at subatmospheric pressures where buoyancy induced convection is minimized. These observations clearly show the diffiusion/convection controlled reaction of the gas-phase homogereous combustion of $\mathrm{Mg}$.

A comparison of the burning behavior of $\mathrm{Mg}$ samples in the normal gravity tests at 0.1 atm versus the tests performed at $0.01 \mathrm{~g}$ at $1 \mathrm{~atm}$ was not possible. Although according to the $p^{2} g$ relationship the same buoyant conditions an be simulated under the two experimental conditions, the distorting effect of gravity on the shape of the molten metal sample at normal gravity can not be eliminated. The cylindrical shape of the $\mathrm{Mg}$ sample is rapidly destroyed after melting leading to a total collapse of the sample at $1 \mathrm{~g}$. In addition, no combustion event is detected at $0.1 \mathrm{~atm}$. Instead, the metal is violently evaporated when, at the boiling temperature of $\mathrm{Mg}$, the force generated by its vapor pressure exceeds the structural integrity of the surface oxide coating leading to its rupture. A nonexplosive surface reaction on the finely divided condensing metal particles appears to take place afterward leaving small oxide particies scattered around the combustion chamber. This dust is a combination of $\mathrm{Mg}, \mathrm{MgO}$, and some undetermined intermodiate oxides. This behavior may be due to a switch from a homogeneous gas-phase reaction to a heterogeneous surface reaction in the particles surface at sufficiently low pressures. The possibility of $\mathrm{Mg}-\mathrm{O}_{2}$ heterogeneons reactions occurring in the oxide surface at low temperatures and pressures has been proposed by Markstein in a study of the reaction of small metal particles externally beated at very low pressures ${ }^{20}$. 


\section{Conclusions}

The combustion behavior of metal-oxygen reactions in a weakly buojant environment is sudied to understand the rate-controlling mechanisms in the homogeneous and heterogeneous combustion of bulk metals. The experimental setup consists of a 1000-W xenon lamp that irradiates the top surface of cylindrical titanium and magnesium specimens, $4 \mathrm{~mm}$ in diameter and $4 \mathrm{~mm}$ in length, in a quiescent, pure-oxygen earvironment at pressures ranging from 0.1 to $4.0 \mathrm{~atm}$. Reduced gravity is obtained from an aircraft flying parabolic trajectories. Qualitative observations, propagation rates and buming times are extracted from high-speed cinematography. Emission spectra of gasphase reactions are obtained with an imaging spectrograph/diode array system.

A weakly buoyant environment is generated at low pressures under normal gravity $(1 \mathrm{~g})$ and also at a pressure of $1 \mathrm{~atm}$ under reduced gravity $(0.01 \mathrm{~g})$. The similarity between these two experimental conditions comes from the $p^{2} g$ factor extracted from the Grashof number. Lower propagation velocities of the molten interface on titanium samples (heterogeneons surface burning) are found at progressively lower pressures at I 8 . In the reduced gravity experiments at $1 \mathrm{~atm}$, the propagation velocity is almost balf the value obtained at $1 \mathrm{~g}$. As expected from the $p^{2} g$ broyancy scale, the propagation velocity at 0.1 atm and $1 \mathrm{~g}$ is relatively close to the valoe found at $1 \mathrm{~atm}$ and $0.01 \mathrm{~g}$. These rates are compared to theoretical results from heat conduction analyses with a diffusion/convection controlled reaction. The close agreement found between experimental and theoretical values indicate the importance of natural convection-enhanced oxygen transport on combustion rates.

For magnesium samples (homogeneous gasphase burning), progressively longer burning times are experienced at lower pressures and $1 \mathrm{~g}$. Under reduced gravity condtions at $1 \mathrm{~atm}$ a burning time twice as long as in $1 g$ is exhibited. Eowever, in this case the validity of the $p^{2} g$ buoyancy scale remains untested due to the inability to obtain steady gas-phase burning of the magnesium sample at $0.1 \mathrm{~atm}$; at this pressure the metal sample simply evaporates without ignition. followed by which appears as nonexplosive surface reaction on the finely divided condensing metal particles. Nevertheless, a clear trend of longer buening times and larger flame standoff distances at low pressures and at low gravity points to a diffusion/conpection controlled reaction. An unique aspect of this work is the use of a reduced gravity environment to evaluate the role of buoyancy-induced oxidizer transport in the burning rate of bulk metal- oxygen reactions at a constant pressure. This tochnique eliminates the uncertainty introduced by the influence of other possible pressure-dependent ratecontrolling mechanisms in studies of weakly buoyant metal flames at low pressures.

\section{Acknowledgments}

This work is supported by the National Aeronautics and Space Administration under Grant NASA-NAG3-1685. The authors gratefully acknowledge the technical supervision of Robert Friedman from the NASA Lewis Research Center.

\section{References}

1. Mortazavi, S., Sunderland, P. B., Jung, J., and Faeth, G. M. Second International Microgravity Combustion Workshop, NASA Conference Publication 10113, pp. 107-112, NASA Lewis Research Center, Cleveland, 1993.

2. Chan, W. and Tien, J., Combust. Sci. and Technol., 18, 139-143 (1978).

3. Ross, H. D., Sotos, R G., and Tien, J., Combust. Sci. and Technol., 75, 155-160 (1991).

4. Yuan, T., Durox, D., and Villermawx, E., Combust. Sci. and Technol., 92, 69-86 (1993).

5. Hirano, T., Sato, $\mathbf{K}$., Sato, $Y$., and Sato, J., Combust. Sci. and Technol., 32, 137-159 (1983).

6. Kirschfeld, L., Angewondte Chemie, 71, 663-667 (1959).

7. Harrison, P. L. and Yoffe, A. D., Proc. Royal Soc., London, 261A, 357-370 (1961).

8. Steinberg, T. A, Wilson, D. B., and Benz, F. J., in Flammability and Sensitivity of Materials in Oxygen-Enriched Atmospheres: Sixth Volume, STP 1197 (Janoff, D. D. and Stoltzfus, J. M., Ed5.), American Society for Testing and Materials, 133-145, Philade]phia 1993.

9. Brzustowsici, T. A and Glassman, I., in Heterogeneous Combustion, AIAA Series in Progress in Astronautics and Aeronautics, Vol. 15 (Wolthard, H. G., Glassman, I., and Green, L., Jr., Eds.), Academic Press, 117-158, New York, 1964. 
Copyright $\odot$ 1997, American Institute of Aeronautics and Astronautics, Inc.

AIAA-98-0570

10. Abbud-Madrid, A, Branch, M. C., and Daily, J. W., Twenty-Sixth Symposium (International) on Combustion. The Combustion Institute, 19291936, Pittsburgh, 1996.

11. Abbud-Madrid, A., The Influence of Gravity on the Ignition and Combustion of Bulk Titanium and Magnesium, Ph.D. Dissertation, University of Colorado at Boulder, University Microfilms, Inc., 9717551, Ann Arbor, 1996.

12. Leibowitz, L., Baker, L. Jr., Schnizlein, J. G., Mishler, L. W., and Bingle, J. D., Nucl. Scl. and Eng., 15, 395-403 (1963).

13. Hirano, T., Sato, Y., Sato, K., and Sato, J., Oxid. Comm., 6, 113-124 (1984).

14. Stcinberg. T. A and Benz, F. J., in Flammability and Sensitivity of Materials in Oxygen-Enriched Atmospheres: Fifh Volume, STP 1111 (Stoltzfus, J. M., and Mellroy, K., Eds.), American Society for Testing and Materials, Philadelphia, 1991.

15. Kofstad, P., High Temperature Corrosion, Elsevier, New York, 1988.

16. Pearson, J. R A., "On Convection Cells Induced by Surface Tension," J. Fluld Mech., 4, 489-500 (1958).

17. Glassman, I and Papas, P., J. Mater. Synth. and Process., 2, 151-159 (1994).

18. Glassman, I., Mellor, A. M, Sullivan, H. F., and Laurendeam, N. M, AGARD Conference Proceedings, No. 52, Paper 19, 1970.

19. The Microgravity Combustion Branch, Microgravity Combustion Science: 1995 Program Update," NASA TM-106858, NASA Lewis Research Center, Cleveland, 1995.

20. Markstein, G. H., Eleventh Symposium (International) on Combustion, pp. 219-234, The Combustion Institute, Pittsburgh, 1967 
